\title{
High Frequency and Intensity of Drinking may Attenuate Increased Inflammatory Cytokine Levels of Major Depression in Alcohol-use Disorders
}

\author{
Sudan P. Neupane ${ }^{1}$ Lars Lien, ${ }^{2,3}$ Priscilla Martinez, ${ }^{4}$ Pål Aukrust, ${ }^{5,6,7,8}$ Thor Ueland, ${ }^{6,9}$ \\ Tom E. Mollnes, ${ }^{8,10,11}$ Knut Hestad $^{2,12}$ \& Jørgen G. Bramness ${ }^{1,13}$ \\ 1 SERAF-Norwegian Centre for Addiction Research, University of Oslo, Oslo, Norway \\ 2 Innlandet Hospital Trust, Hamar, Norway \\ 3 Department of Public Health, Hedmark University College, Elverum, Norway \\ 4 Alcohol Research Group, University of California, Berkeley, CA, USA \\ 5 Research Institute for Internal Medicine, Oslo University Hospital, Rikshospitalet, Oslo, Norway \\ 6 Section of Clinical Immunology and Infectious Diseases, Oslo University Hospital, Rikshospitalet, Oslo, Norway \\ 7 Institute of Immunology, University of Oslo, Oslo, Norway \\ 8 K.G. Jebsen Inflammatory Research Center, University of Oslo, Oslo, Norway \\ 9 Institute of Clinical Medicine, University of Oslo, Oslo, Norway \\ 10 Department of Immunology, Oslo University Hospital Rikshospitalet, University of Oslo, Oslo, Norway \\ 11 Research Laboratory Nordland Hospital, Bodø and Faculty of Health Sciences, University of Tromsø, Troms $\varnothing$, Norway \\ 12 Department of Psychology, The Norwegian University of Science and Technology, Trondheim, Norway \\ 13 Department of Pharmacoepidemiology, Norwegian Institute of Public Health, Oslo, Norway
}

\section{Keywords}

Alcohol; Comorbidity; Cytokines; Depression; Immune activation; Nepal.

\section{Correspondence}

Sudan P. Neupane, M.B.B.S., M.Phil., Ph.D.

Research fellow, Norwegian Centre for

Addiction Research (SERAF), University of

Oslo, Post box 1039, Blindern, N-0315 Oslo,

Norway.

Tel.: +47 23368980 ;

Fax: +47 23368986 ;

E-mail: s.p.neupane@medisin.uio.no

Received 21 March 2014; revision 2 June

2014; accepted 3 June 2014

\begin{abstract}
SUMMARY
Aims: As major depression (MD) is often comorbid with alcohol-use disorders (AUD) and alcohol itself modulates the immune system, we examined serum levels of interleukin (IL)-6, IL-10, tumor necrosis factor (TNF), and interferon (IFN) $-\gamma$ in AUD patients with and without MD. Putative interactions between alcohol variables and MD on cytokine levels were also assessed. Methods: A consecutive sample of inpatients with AUD ( $N=176)$ from eight alcohol treatment centers in Kathmandu, Nepal, was assessed for alcohol use and depression by administering fully structured psychiatric interviews. Serum cytokine levels were determined using multiplex technology. Results: Alcohol-use disorders patients with a positive history of MD had higher levels of the inflammatory cytokines IL-6 $(P=0.019)$, TNF $(P=0.020)$, and IFN- $\gamma(P=0.001)$, but not of IL-10 $(P=0.853)$. AUD patients with MD had higher concentrations of cytokines compared with those without, regardless of the severity of the alcohol problem, but the difference was greater among those drinking in lower frequency and intensity. Conclusion: These findings provide evidence for altered functioning of the immune system in AUD patients with comorbid MD. However, frequent and intense drinking may attenuate the difference in the cytokine profiles between AUD patients with and without MD.
\end{abstract}

\section{Introduction}

Major depression (MD) is a highly prevalent and potentially debilitating psychiatric illness characterized by sustained depression of mood, anhedonia, significant changes in weight, psychomotor disturbance, fatigue, inability to concentrate, inappropriate guilt, as well as recurrent thoughts of death $[1,2]$. Recent advances in psychoneuroimmunology have demonstrated that about a third of depressed individuals exhibit activated inflammatory responses as measured by increased circulating levels of inflammatory cytokines such as interleukin-6 (IL-6), tumor necrosis factor (TNF), and interferon gamma (IFN- $\gamma$ ) [3-5]. It has been postulated that the activation of these inflammatory mediators directly contributes to the development of depressive disorders [6]. Evidence shows enhanced inflammation can provoke a depressive episode [7], cause depressive symptoms as happens with IFN- $\alpha$-based immunotherapy [8], drive an ongoing depressive state or modulate the severity of depressive symptoms [9], and persist after clinical recovery [10].

Approximately $30 \%$ of patients with MD will satisfy a DSMIV-TR diagnosis of alcohol-use disorder (AUD: alcohol abuse or alcohol dependence) in their lifetime [11]. Even higher prevalence of MD is reported among patients with AUD in different populations [12-14]. For example, $45 \%$ of Nepalese inpatients 
with AUD would satisfy a lifetime diagnosis of major depressive episode [15].

Alcohol modulation of the immune function has been evidenced by altered levels of various cytokines in plasma, liver, lungs, and brain samples in patients with AUD [16,17]. While acute and moderate use of alcohol has been associated with down-regulated inflammatory responses characterized by inhibited TNF and IL- 6 and augmented IL-10 production, chronic heavy alcohol use results in increased inflammatory responses [18]. Although reports in the literature vary according to the cytokine studied, the severity of alcohol problems, and hepatic damage, it is clear that alcohol-use disorders result in dysregulated cytokine balance [19].

Given the individual effect of alcohol use and depression on immune function and the frequent comorbidity between AUD and $\mathrm{MD}$, the effect of comorbidity on immune function as a common underlying biological mechanism requires further investigation. Relatively few studies have examined immune activation among alcohol-dependent individuals; even fewer have made direct comparisons between AUD patients with and without depression. Furthermore, the few studies that have made such comparisons lacked measurements of circulating cytokines and had small sample sizes $(<100)$. Using ex vivo measures of immune cell function, Schleifer et al. [20] observed that enumerative and functional immune changes associated with depression among patients with AUD were consistent with those seen in depression alone. The authors also identified associations between attributes such as smoking and recency of alcohol intake with immune changes in alcohol use and, more modestly, in depression. Relatedly, other studies have identified ethnic and socio-demographic differences in the circulating cytokine profile of people with depression and alcohol misuse in both clinical and community samples [21,22]. Thus, it is important to include relevant behavioral attributes and to examine populations from diverse settings in studies of the theoretical underpinnings of the relationship between AUD, depression, and inflammation.

We hypothesized that MD comorbid with AUD would result in further increases of the inflammatory cytokines and that alcohol severity would relate directly to their levels. To study this hypothesis, we measured serum levels of four cytokines that have been related to immunodysregulation in these disorders (i.e., IL-6, IL-10, TNF, and IFN- $\gamma$ ), in a Nepalese sample of AUD inpatients with and without MD.

\section{Materials and methods}

\section{Recruitment Procedure and Data Collection}

This article is based on cross-sectional data of Nepalese patients in eight alcohol treatment centers in Kathmandu, Nepal. All institutions but one used a 12-step-based rehabilitation program for recovery. The study's general methodology has been described previously [15]. Participants were free from alcohol for at least 4 days before the collection of blood samples, and psychiatric interviews were held at least 10 days after enrollment into treatment. The study protocol was approved by the relevant Norwegian and Nepalese ethics committees prior to data collection.
We approached 221 consecutively admitted clients, explained the study procedure and invited them to participate. After screening for inclusion, 204 eligible participants provided written informed consent (thumb print with witness's signature from illiterate clients) together with a separate consent for collection of a blood sample. There were 188 patients who satisfied the DSMIV-TR criteria for an alcohol-use disorder ( 24 alcohol abuse and 164 alcohol dependence); 11 participants refused to offer blood samples. The study comprised of the remaining 176 patients with AUD.

A general physical examination was carried out, and about $8 \mathrm{~mL}$ of antecubital venous blood was collected on a BD vacutain$\mathrm{er}^{\circledR}$ gel tube in the late afternoon. Within $2.5 \mathrm{~h}$ of sampling, the serum was separated on a swing-out centrifuge $(1300 \times g)$ at room temperature and transferred into a polypropylene tube, which was then stored at $-70^{\circ} \mathrm{C}$. Using solidified carbon dioxide, the frozen samples were transported to the research laboratory at Nordland Hospital in Bodø, Norway, where the cytokine assays were performed.

\section{Questionnaires}

Psychometric evaluation was performed using the World Health Organization's second version of the Composite Interview Diagnostic Interview (CIDI; WHO, 1994) administered in the local language. The DSM-IV-TR diagnoses of alcohol-use disorders (305.00 alcohol abuse or 303.90 alcohol dependence) and major depressive episodes (MD) were obtained from the CIDI. Patients were asked to denote the time at which the cardinal symptoms of MD appeared and lasted for at least 2 weeks, excluding recent complaints. A modified version of the demographic module of the CIDI was used to record the variables gender (female/male), place of residence (rural/urban), educational level (illiterate or primary/ higher), marital status (married/other), caste category (high/low), employment (regular income source/other), and social support (inadequate/adequate). Alcohol-use parameters were measured using the Alcohol Use Disorder Identification Test (AUDIT) [23]. We also administered the Hopkins Symptoms Checklist- 25 (HSCL-25) [24] to evaluate psychological distress in the last 15 days that would capture transient mood alterations in the recently detoxified AUD sample. Recent distress was defined as average HSCL-25 score of 1.75 or higher in both genders. The instruments have shown acceptable reliability and were locally or regionally validated, and their translated Nepali versions have been used previously [25-27].

\section{Determination of Cytokine Levels}

Serum levels of the three inflammatory cytokines IL-6, TNF, and IFN- $\gamma$, and an anti-inflammatory cytokine IL-10 were assayed by a multiplex cytokine assay (Human Bio-Plex; Bio-Rad Laboratories Inc., Hercules, CA, USA) according to instructions from the manufacturer. The interassay and intraassay coefficients of variation were $<10 \%$ for all analyses. All values were within the standard curve, except for two extreme outliers for TNF, which were excluded. Additionally, serum levels of gamma-glutamyl transferase $(\gamma-G T)$, a general marker for liver injury, and carbohydrate deficient transferrin (CDT), a useful marker for recent alcohol 
consumption, were determined using standard photometry and capillary electrophoresis, respectively.

\section{Statistical Analysis}

SPSS version 20 for Windows (SPSS Inc., Chicago, IL, USA) was used for the statistical analysis of the data. Descriptive statistics are presented as percentages, means, and standard deviations. The association between demographics and MD diagnosis was observed by Pearson's chi square-test. The spread of the cytokine values were rather skewed and therefore reported by categories of alcohol variables as medians and 25/ 75 percentiles. Their distributions across no-MD and MD groups are shown in boxplots.

The nonparametric Mann-Whitney $U$-test was used to observe differences in cytokine concentrations according to demographic and alcohol-use parameters. For ease of presentation, weekly frequency of alcohol use, ethanol consumed per drinking day, and abstinence duration were dichotomized at 4 days, 10 standard units, and 10 days, respectively. Spearman's correlation was used for estimating correlations between cytokines and other continuous variables. Finally, the role of alcohol variables in the relationship between MD and cytokines is depicted as bar diagrams. All tests were two-tailed with the statistical significance set at 5\% level.

\section{Results}

\section{Patient Characteristics and Cytokine Profile}

The study sample had a mean age of $35.5( \pm 10.2)$ years with a history of habitual drinking of alcohol for $16.9( \pm 10.0)$ years and abstinence from alcohol for $33.8( \pm 22.5)$ days prior to interview. As many as $78 \%$ of the current sample had a total AUDIT score of above 20 (mean 27.9 [SD 8.7]), which according to WHO indicates high likelihood for meeting alcohol dependence diagnosis. It consisted predominantly of males $(89 \%)$ and urban residents $(69 \%)$. Table 1 lists the socio-demographic and clinical characteristics of the sample by depression history. Compared with the group that reported no history of MD, MD patients ( $\mathrm{n}=80,45 \%$ including $36 \%$ who had MD in the 12 -months preceding assessment) were less likely to be cohabitating or to have a regular source of income. The two groups did not vary by the duration, frequency and extent of alcohol use, abstention duration in current treatment, or recent medication (Table 1).

There were no significant differences in cytokine levels across socio-demographic variables, such as, gender, age group, marital status, education, income level, place of residence, and social support or the month of sampling (data not shown).

\section{Serum Cytokine Levels in Depression}

We first compared cytokine levels in AUD patients with and without a history of MD. The MD group had increased levels of the inflammatory cytokines IL-6 $(P=0.019)$, TNF $(P=0.020)$, and IFN- $\gamma(P=0.001)$, but not of IL-10 $(P=0.853)$ compared with the no-MD group (Figure 1). Satisfying an MD diagnosis in the
Table 1 Demographic and medical characteristics of alcohol-use disorder inpatients $(N=176)$ in Nepalese alcohol treatment centers by their lifetime history of major depression (MD)

\begin{tabular}{|c|c|c|c|c|c|}
\hline \multirow[b]{2}{*}{ Characteristic } & \multicolumn{2}{|c|}{$\begin{array}{l}\text { No MD } \\
(\mathrm{n}=96)\end{array}$} & \multicolumn{2}{|c|}{$\begin{array}{l}\mathrm{MD} \\
(\mathrm{n}=80)\end{array}$} & \multirow[b]{2}{*}{$P$-value* } \\
\hline & $n$ & $\%$ & $\mathrm{n}$ & $\%$ & \\
\hline Gender: Male & 87 & 91 & 69 & 86 & 0.475 \\
\hline Residence: Urban & 33 & 34 & 22 & 28 & 0.414 \\
\hline $\begin{array}{l}\text { Education: illiterate or primary } \\
\text { level (vs. higher) }\end{array}$ & 31 & 32 & 21 & 26 & 0.411 \\
\hline Marital status: married & 69 & 72 & 40 & 50 & 0.003 \\
\hline Hindu caste: high & 53 & 55 & 43 & 54 & 0.880 \\
\hline $\begin{array}{l}\text { Employment: regular source } \\
\text { of income }\end{array}$ & 65 & 68 & 39 & 49 & 0.009 \\
\hline Social support: adequate & 70 & 73 & 55 & 69 & 0.617 \\
\hline $\begin{array}{l}\text { Institution: rehabilitation } \\
\text { center (vs. hospital) }\end{array}$ & 82 & 85 & 70 & 88 & 0.826 \\
\hline Medication: any use in the past week & 31 & 32 & 33 & 41 & 0.271 \\
\hline Drinking frequency: $\geq 4$ days weekly & 69 & 73 & 61 & 77 & 0.600 \\
\hline Daily drinking units: average $\geq 10$ units & 63 & 76 & 56 & 74 & 0.850 \\
\hline
\end{tabular}

No MD and MD cases for some variables may not sum to 176 due to missing data. ${ }^{*} P$-value for the difference between never depressed and ever depressed group, by chi square-test. Significant values are shown in bold.

preceding 12-month period showed results similar to lifetime MD, but recent distress was not related to higher cytokine levels. In fact, MD patients without recent psychological distress had significantly higher levels of TNF $(P=0.012)$ and IFN- $\gamma(P=0.001)$ compared with non-MD patients without psychological distress. This difference was not seen in recent distress.

\section{Alcohol-related Measures}

Some alcohol-use variables were related to cytokine levels (Table 2). High-frequency drinking ( $\geq 4$ days per week) was related to higher serum IFN- $\gamma(P=0.026)$ and IL-10 $(P=0.005)$ levels compared with the group who drank less frequently. Similarly, drinking 10 or more standard units of alcohol on a typical day was related to higher IL-10 $(P=0.024)$ levels compared with drinking moderately. The cytokines did not differ across categories of AUD (alcohol abuse and alcohol dependence) and abstinence duration (below 10 days and above; Table 2). None of the studied cytokines correlated significantly with $\gamma$-GT and CDT levels. Abusing an illicit substance in the preceding year did not influence cytokine levels $(P>0.05$ for all cytokines). However, current smoking was associated with lower IL-6 level, particularly in the MD group $(P=0.010)$.

\section{Interaction between Alcohol and Depression Measures}

The interaction between alcohol and depression measures is shown in Figure 2. Generally, AUD patients with a positive history of MD tended to have higher concentrations of cytokines 
Figure 1 Boxplot showing median (central lines), $25 \%$ and $75 \%$ quartile ranges around the median (box width), and upper and lower limits (whiskers) of serum levels of interleukin-6 (IL-6), tumor necrosis factor (TNF), interferon gamma (IFN- $\gamma$ ), and IL-10 in alcohol-use disorder patients reporting no history of lifetime major depression (no MD, $n=96$ ) or reporting a lifetime history of major depression (MD, $\mathrm{n}=80$ ). . -values were calculated by MannWhitney U-test.
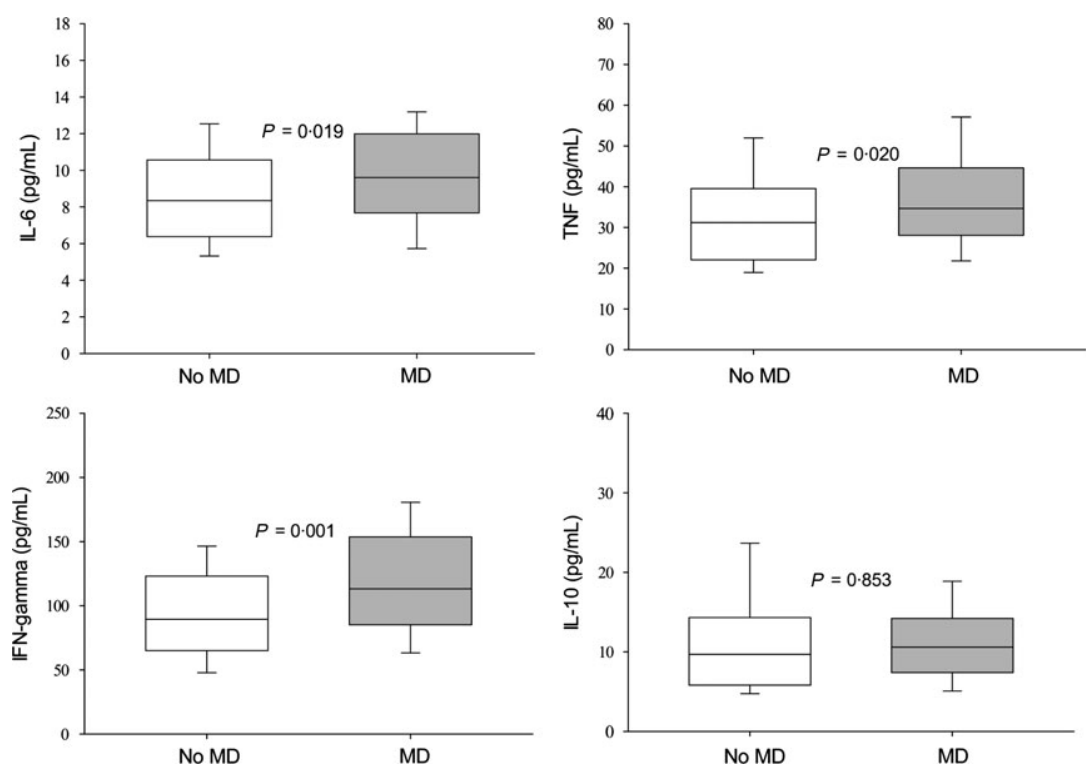

compared with those without MD, regardless of the severity of alcohol problems. Compared with patients without a history of major depression, MD patients who used alcohol 3 days or fewer per week in quantities below 10 standard units had significantly higher IL-6, TNF, IFN- $\gamma$, and IL-10 levels. However, this association was attenuated in the groups who drank with higher frequency and intensity. Abstinence duration dichotomized at 10 days did not show a clear trend. Nonetheless, levels of IL- 6 and IFN- $\gamma$ were higher among MD patients with more than 10 days of abstinence.

\section{Discussion}

This cross-sectional study from Nepal investigated the relationship between MD and cytokines specifically in alcohol-use disorder (AUD) patients. Patients with comorbid MD showed increased levels of the inflammatory cytokines IL- 6 , TNF, and IFN- $\gamma$ relative to the AUD patients without MD comorbidity, replicating earlier findings from non-AUD populations [3,4]. The impact of MD on immune responses was strong in AUD patients with less heavy alcohol use (i.e., drinking with lower frequency and amounts, and satisfying fewer symptoms of AUD), and these patients also showed increased IL-10 levels. Patients with more heavy alcohol use; however, had a nonsignificant difference in cytokine levels between MD and non-MD groups. This indicates that heavy alcohol use may attenuate the difference in cytokine profiles between AUD patients with and without MD. Thus, our findings lend support to the hypothesis that AUD and MD interact in a complex manner and that immune mechanism may be a connecting piece of the puzzle [28].

Patients with MD as a comorbidity have been found to have raised plasma and serum levels of inflammatory markers as compared with patients without this comorbidity in a multitude of medical conditions, including cardiovascular disease [29], multiple sclerosis [30], cancer [31], and fibromyalgia [32].
Patients with addiction problems are important groups to consider in this context because of the high rates of comorbidity with MD with potential impact on immunity. Patients with AUD are of particular interest as alcohol itself acts as a modulator of the immune system, and its chronic use has been reliably demonstrated to activate both innate and adaptive immunity $[17,18]$. However; unlike in depression disorders, immune alterations in alcohol use have been less clear and often linked to associated medical morbidities, liver damage, and smoking status [33]. The cytokine response in AUD could potentially reflect liver damage, but in this study, we found no association between cytokine levels and $\gamma$-GT, a general marker of alcoholinduced liver disease. Neither was the marker for recent alcohol use (CDT) related to the cytokine levels. Smoking history, nonetheless, was associated with cytokine alterations. Patients with MD as a comorbidity had increased serum levels of IL-6, TNF, and IFN- $\gamma$, but not IL-10 as compared to those without MD, suggesting an enhanced inflammatory phenotype in patients with the co-existence of AUD and MD. We also found that the association between inflammatory cytokines and depression among people with AUD was less obscure when lifetime prevalence was considered as opposed to past-year major depressive episodes or recent distress. This may reflect that the inflammatory subset of depression in AUD is also pervasive as a trait ingredient of the comorbidity and in a lesser degree, a time-dependent association in relation to recent depressive episodes. Indeed, some evidence supports the lingering nature of immune activation in depressive disorders $[10,34]$. These issues as well as whether the increased inflammatory markers predict future episodes of depression in AUD should be explored with prospective studies. If cytokine profiles or changes in profiles are consistently observed in longitudinal studies to influence the onset or remission of depression in AUD, interventions targeting immune function may be effective and should be developed and tested. 


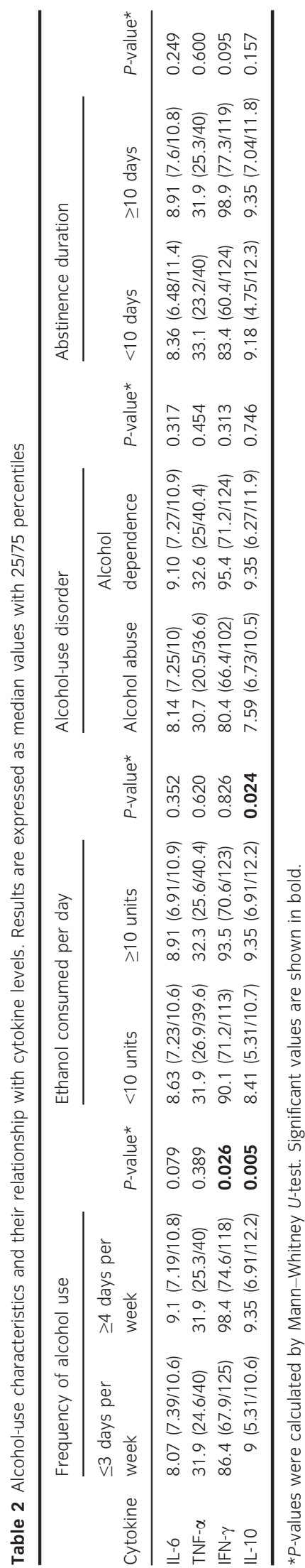

In AUD patients with heavy alcohol use, the impact of MD on cytokine levels was attenuated. Moreover, patients with a higher frequency of alcohol intake ( $\geq 4$ days per week) had higher IFN- $\gamma$ levels than the other individuals with AUD. The bulk of the literature on alcohol-induced immune activation is based on experiments in animal models and in vitro studies. Based on such studies, it has been suggested that regulation of inflammatory cytokine production by monocyte and macrophage seems to be depending on the length of alcohol treatment [17]. Our findings in this study suggest that proxy measures of AUD severity, such as ethanol intake with high frequency and intensity, may influence the cytokine levels in serum. In heavy drinkers, this may be the dominant factor in relation to inflammation even in patients with accompanying MD. In those drinking with lower frequency and amounts, further increased levels of inflammatory cytokines in MD (compared with the more heavy drinkers) may be the result of reduced tryptophan and increased kynurenine levels, as evidenced in short-term alcohol exposure in humans [35]. This issue and how alcohol abuse in terms of frequency, quantity, and type of beverage impacts on the cytokine and T-cell homeostasis merit further investigation.

The anti-inflammatory cytokine IL-10 did not vary significantly between groups with and without MD. In contrast, high levels of alcohol-use were significantly related to circulatory IL-10 levels. Thus, drinking $\geq 10$ units of pure alcohol on a typical day and drinking $\geq 4$ days per week were both associated with high IL-10 level. It may be that IL-10-mediated anti-inflammatory responses in conjunction with increased IL-6 levels reflect activation of the Th2 T-cell subset in these patients [36]. Hence, although decreased IL-10 responses have been associated with alcoholinduced liver disease, our findings suggest that severe alcohol abuse may be associated with increased IL-10, at least in the context of accompanying MD. This could potentially promote immunosuppression in these individuals.

We acknowledge some limitations to our study. Because of the cross-sectional nature of the study, without a control sample, we are unable to draw a conclusion about whether the increase in the levels of cytokines was actually associated with the disorders considered and not featured artifacts of other inflammatory processes in these patients. We did not observe any variation in our findings of cytokine difference across MD and alcohol characteristics according to gender or type of treatment being received. However, it is important to control for antidepressant and anti-inflammatory medications, as these can clearly confound the results [37]. Moreover, serum levels may not necessarily reflect the levels within the microenvironment such as the brain. In spite of these limitations, this study provides important immunological insights into the complex interactions between the frequently comorbid conditions of alcohol and depression disorders.

In conclusion, the results from this study suggest that a treatment sample of alcohol-use disorder patients with positive history of major depression has increased levels of circulatory IL-6, TNF, and IFN- $\gamma$, but not of IL-10. However, these differences seem to attenuate in heavy alcohol use. Prospective, controlled studies are needed to confirm the role of alcohol in the relationship between depression and immunity, vis-à-vis the potential role of cytokines in the alcohol-depression comorbidity. 

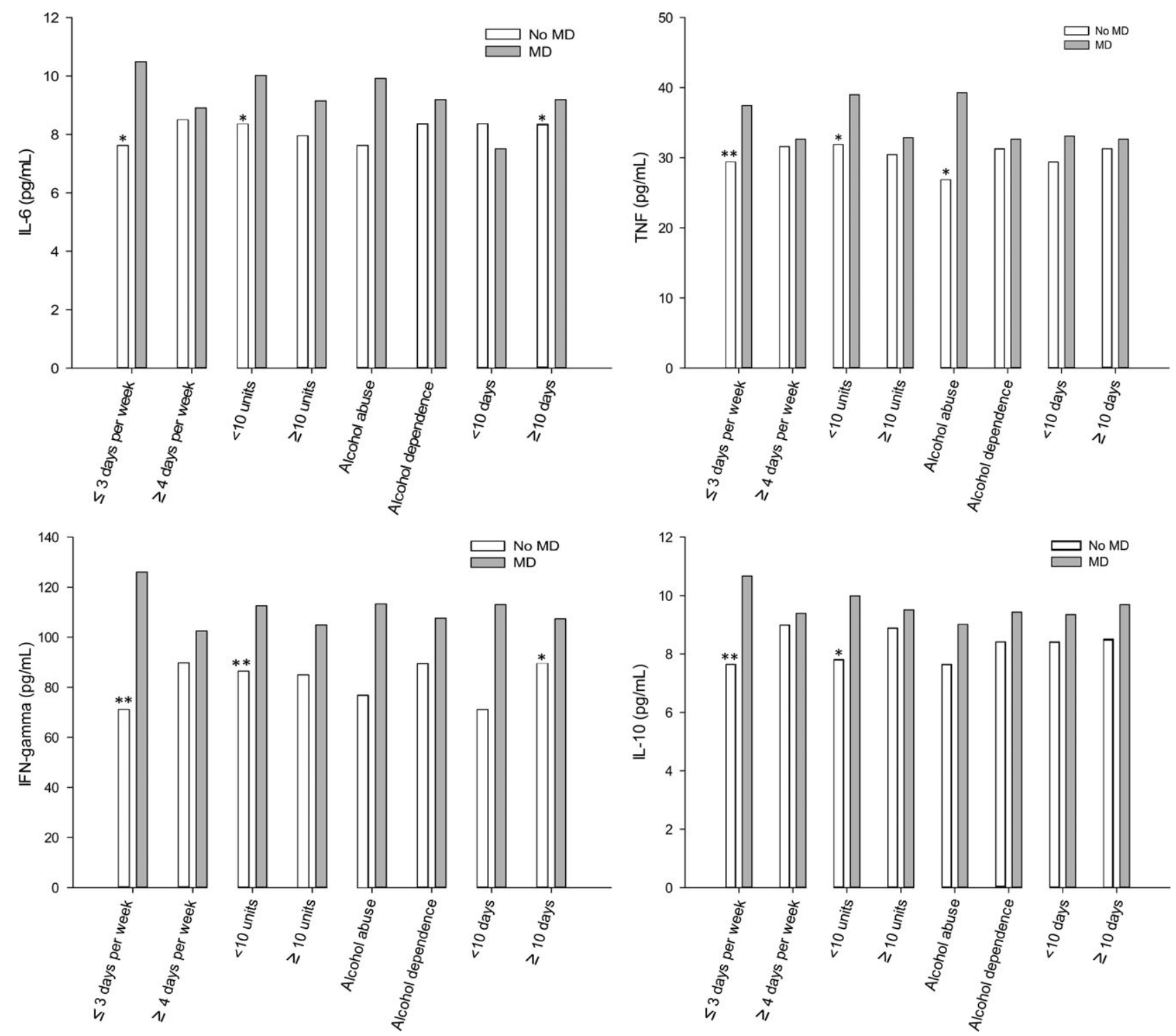

Figure 2 Serum levels of interleukin-6 (IL-6), tumor necrosis factor (TNF), interferon gamma (IFN- $\gamma$ ), and IL-10 in alcohol-use disorder patients reporting no history of lifetime major depression (no $M D, n=96$ ) or reporting a lifetime history of major depression (MD, $n=80$ ) by drinking frequency, intensity, alcohol abuse or dependence and abstinence duration. Data are presented as medians. Significant differences in the no MD and MD groups by the severity of alcohol-use features, calculated by Mann-Whitney $U$-test, are indicated by asterisk marks $(* P<0.05, * * P<0.01)$.

\section{Acknowledgments}

The authors would like to thank the treatment centers and their clients for their participation in this research.

\section{Conflict of Interest}

The authors declare no conflict of interest.

\section{References}

1. Mathers C, Fat DM, Boerma JT. The global burden of disease: 2004 update. Zeneva: World Health Organization, 2008.

2. American Psychiatric Association. Diagnostic and statistical manual of mental disorders, 5 th edn. Washington, DC: American Psychiatric Association, 2013.

3. Dowlati Y, Herrmann N, Swardfager W, et al. A meta-analysis of cytokines in major depression. Biol Psychiatry 2010;67:446-457.

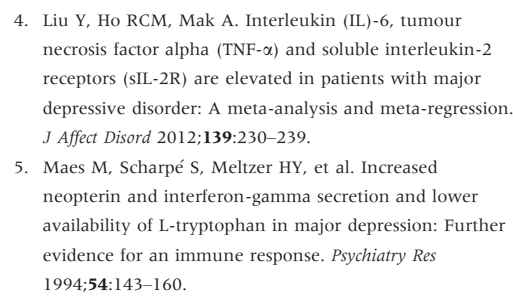

4. Liu Y, Ho RCM, Mak A. Interleukin (IL)-6, tumour necrosis factor alpha (TNF- $\alpha$ ) and soluble interleukin-2 receptors (sIL-2R) are elevated in patients with major depressive disorder: A meta-analysis and meta-regression. $J$ Affect Disord 2012;139:230-239.

5. Maes M, Scharpé S, Meltzer HY, et al. Increased neopterin and interferon-gamma secretion and lower availability of L-tryptophan in major depression: Further evidence for an immune response. Psychiatry Res 1994;54:143-160

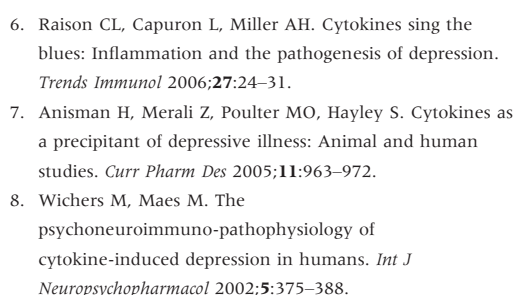


9. Motivala SJ, Sarfatti A, Olmos L, Irwin MR Inflammatory markers and sleep disturbance in major depression. Psychosom Med 2005;67:187-194.

10. Raedler TJ. Inflammatory mechanisms in major depressive disorder. Curr Opin Psychiatry 2011;24:519525.

11. Sullivan LE, Fiellin DA, O'Connor PG. The prevalence and impact of alcohol problems in major depression: A systematic review. Am J Med 2005; 118:330-341.

12. Grant BF, Stinson FS, Dawson DA, et al. Prevalence and co-occurrence of substance use disorders and independent mood and anxiety disorders: Results from the National Epidemiologic Survey on Alcohol and Related Conditions. Arch Gen Psychiatry 2004;61:807-816.

13. Lynskey MT. The comorbidity of alcohol dependence and affective disorders: Treatment implications. Drug Alcohol Depend 1998;52:201-209.

14. Swendsen JD, Merikangas KR, Canino GJ, Kessler RC Rubio-Stipec M, Angst J. The comorbidity of alcoholism with anxiety and depressive disorders in four geographic communities. Compr Psychiatry 1998;39:176-184.

15. Neupane SP, Bramness JG. Prevalence and correlates of major depression among Nepalese patients in treatment for alcohol-use disorders. Drug Alcohol Rev 2013;32:170177.

16. Goral J, Karavitis J, Kovacs EJ. Exposure-dependent effects of ethanol on the innate immune system. Alcohol 2008;42:237-247.

17. Crews FT, Bechara R, Brown LA, et al. Cytokines and alcohol. Alcohol Clin Exp Res 2006;30:720-730.

18. Szabo G, Mandrekar P. A recent perspective on alcohol immunity, and host defense. Alcohol Clin Exp Res 2009:33:220-232
19. Achur RN, Freeman WM, Vrana KE. Circulating cytokines as biomarkers of alcohol abuse and alcoholism. I Neuroimmune Pharmacol 2010;5:83-91.

20. Schleifer SJ, Keller SE, Czaja S. Major depression and immunity in alcohol-dependent persons. Brain Behav Immun 2006;20:80-91.

21. Irwin M, Miller C. Decreased natural killer cell responses and altered interleukin- 6 and interleukin-10 production in alcoholism: An interaction between alcohol dependence and African-American ethnicity. Alcohol Clin Exp Res 2000;24:560-569.

22. Stowe RP, Peek MK, Cutchin MP, Goodwin JS. Plasma cytokine levels in a population-based study: Relation to age and ethnicity. J Gerontol A Biol Sci Med Sci 2010;65:429-433.

23. Babor TF, Higgins-Biddle JC, Saunders JB, Monteiro MG. The alcohol use disorders identification test: Guidelines for use in primary care, 2nd edn. Zeneva: World Health Organization, 2001

24. Derogatis LR, Lipman RS, Rickels K, Uhlenhuth EH, Covi L. The Hopkins Symptom Checklist (HSCL): A self-report symptom inventory. Behav Sci 1974;19:1-15.

25. Carey KB, Carey MP, Chandra PS. Psychometric evaluation of the alcohol use disorders identification test and short drug abuse screening test with psychiatric patients in India. J Clin Psychiatry 2003;64:767-774.

26. Van Ommeren $M$, Sharma B, Thopa S, et al. Preparing instruments for transcultural research: Use of the translation monitoring form with Nepali-speaking Bhutanese refugees. Transcult Psychiatry 1999;36:285-301.

27. Wittchen HU. Reliability and validity studies of the WHOComposite International Diagnostic Interview (CIDI): A critical review. J Psychiatr Res 1994;28:57-84.
28. Kelley KW, Dantzer R. Alcoholism and inflammation: Neuroimmunology of behavioral and mood disorders. Brain Behav Immun 2011;25(suppl):13-20.

29. Halaris A. Inflammation, heart disease, and depression. Curr Psychiatry Rep 2013;15:1-9.

30. Gold SM, Irwin MR. Depression and immunity: Inflammation and depressive symptoms in multiple sclerosis. Immunol Allergy Clin North Am 2009;29:309-320.

31. Jehn CF, Kuehnhardt D, Bartholomae A, et al Biomarkers of depression in cancer patients. Cancer 2006;107:2723-2729.

32. Bazzichi L, Rossi A, Massimetti G, et al. Cytokine patterns in fibromyalgia and their correlation with clinical manifestations. Clin Exp Rheumatol 2007;25:225-230.

33. Cook RT. Alcohol abuse, alcoholism, and damage to the immune system-a review. Alcohol Clin Exp Res 1998;22:1927-1942.

34. Copeland WE, Shanahan L, Worthman C, Angold A, Costello EJ. Cumulative depression episodes predict later C-reactive protein levels: A prospective analysis. Bio Psychiatry 2012;71:15-21.

35. Badawy AA, Doughrty DM, Marsh-Richard DM, Steptoe A. Activation of liver tryptophan pyrrolase mediates the decrease in tryptophan availability to the brain after acute alcohol consumption by normal subjects. Alcohol Alcohol 2009;44:267-271.

36. Gao B. Hepatoprotective and anti-inflammatory cytokines in alcoholic liver disease. $J$ Gastroenterol Hepatol 2012;27:89-93.

37. Munzer A, Sack U, Mergl R, et al. Impact of antidepressants on cytokine production of depressed patients in vitro. Toxins (Basel) 2013;5:2227-2240. 